\title{
Cerebellar Tuberculoma in a Patient with Lung Tuberculosis New Case: A Case Report
}

\author{
Gemilang Khusnurrokhman*, Tutik Kusmiati \\ Department of Pulmonology and Respiratory Medicine, Faculty of Medicine, Universitas Airlangga/Dr. Soetomo General Hospital, \\ Surabaya, Indonesia.
}

\section{ARTICLE INFO}

Article history:

Received 16 July 2020

Received in revised form 07

September 2020

Accepted 14 September 2020

Available online 30 September 2020

\section{Keywords:}

Lung tuberculosis,

Extra lung tuberculosis,

Central nervous system,

Cerebellar tuberculoma,

Anti-tuberculosis drugs

\begin{abstract}
Background: Tuberculosis (TB) is an infectious disease which can occur in the lungs and extra lung. One kind of extra lung TB is Cerebellum tuberculoma, which is one of rare manifestations form of $\mathrm{TB}$, difficult to diagnosed, because sign and symptom is not specific.

Case: A 17 years-old female presented a TB symptomps. She also had contact with her neighbor that was diagnosed with TB. In physical examination there was reduced motor function on her right extremities. Chest radiology revealed opacities in 2/3 superior in the left lung before treatment. Computed Tomography (CT) head scan with contrast showed ring contrast enhancement in left subcortical hemisphere cerebellum with size as 1,8 $\mathrm{x} 1,8 \times 1,7 \mathrm{~cm}$. Multiple lymphadenopathy in right-left upper-aid jugular and lymph node subcentimeter in right of submandibular, right-left lower jugular with varieties of size, the biggest is $1,6 \times 1,5 \mathrm{~cm}$. After 6 months treatment the tuberculoma of cerebellum was gone.

Conclusion: Cerebellum tuberculoma is a rare presentation of active TB. Radiological imaging plays an important role in the diagnosis and monitoring of the disease. The appereance of tuberculoma on our patient CT showed nodular-enhancing lesion. Intracranial tuberculoma should be treated conservatively, since the majority of these lesions resolve completely with Anti-Tuberculosis Drugs (ATD). Our patient started ATD and the lesions completely disappeared within 6 months. We wanted to present our case due to well-responded to ATD and being an example of atypical presentation of tuberculosis both clinically and radiologically.
\end{abstract}

\section{BACKGROUND}

Tuberculosis (TB) is an infectious disease which can occur in the lungs and extra lung. One kind of extra lung TB is Cerebellar Tuberculoma, which is one of rare manifestation forms of $\mathrm{TB}$ and is difficult to diagnose because the signs and symptoms are not specific. Etiology of TB is Mycobacterium tuberculosis (M.TB). Kruijshaar, et al. in 2004 stated that the incidence of extra lung TB increased in female adolescents of African and Asian races with HIV status. ${ }^{1}$

The incidence of extra lung TB from 2005-2007 had been increased from $14-20 \%$ in $2010-2013$. The prevalence of extra lung TB worldwide is $14 \%$ (6.4 million cases in 2017). ${ }^{2}$ The incidence of extra lung TB in central nervous system was $5-15 \%$. $^{3}$ Tuberculoma in central nervous system should be distinguished from other diagnosis, like tumor or abscess. Tuberculoma is caused by M.TB which is spreading hematogenously. The diagnosis must be made quickly because there are complications such as extremity paralysis and sensory loss. Tuberculoma in central nervous system mostly infected meninges, cerebrum, and rarely in the cerebellum. Arvind, et al. explained that the incidence of cerebellar tuberculoma mostly happens in young age because there is a lot of blood supply. ${ }^{4}$

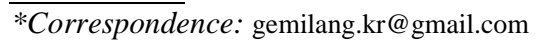




\section{CASE}

A 17-year-old female from Surabaya came with chief complaint of spinning dizziness since 7 days before hospitalized. She also felt right limb weakness, disturbance of consciousness, and nausea. She has history of dry coughing for more than 2 months, and night sweating. She also had contact with her neighbor that was diagnosed with TB. In physical examination, her body mass index was $17.80 \mathrm{~kg} / \mathrm{m}^{2}$ (underweight) and there was reduced motor function on her right extremities (muscle power scale 4 upper and lower right extremities). No abnormality found in other physical examination. No abnormality found in blood examination. The patient had dry cough, there was no sample to exam microbiology for acid fast bacilli smear, phlegm culture, and geneXpert M.TB.

Chest radiology revealed opacities in $2 / 3$ superior in the left lung before treatment (Figure 1A). Chest radiology after 9 months therapy (Figure 1B) and 12 months therapy (Figure 1C). CT head scan with contrast
(Figure 2) showed ring contrast enhancement in left subcortical hemisphere cerebellum with size $1.8 \mathrm{x} 1.8 \mathrm{x}$ $1.7 \mathrm{~cm}$. Multiple lymphadenopathy in right-left upperaid jugular and lymph node sub centimeter in right of submandibular, right-left lower jugular with varieties of size, the biggest was $1.6 \times 1.5 \mathrm{~cm}$. After 6 months of treatment, the cerebellar tuberculoma was gone (Figure $3)$.

On the $7^{\text {th }}$ day of hospitalization, the patient was discharged on ATD $1^{\text {st }}$ cathegory 4 FDC intensive phase for 2 months and injection of streptomycin intramuscular. The patient then continued with phase 2 FDC for 10 months.

\section{DISCUSSION}

Extra lung TB is a manifestation of TB outside of the lungs that could infected pleura, lymph node, abdomen, genitourinary tract, joints, bone, and central nervous system. ${ }^{5}$ Tuberculoma in central nervous system mostly infected meninges, cerebrum, and rarely in the cerebellum.

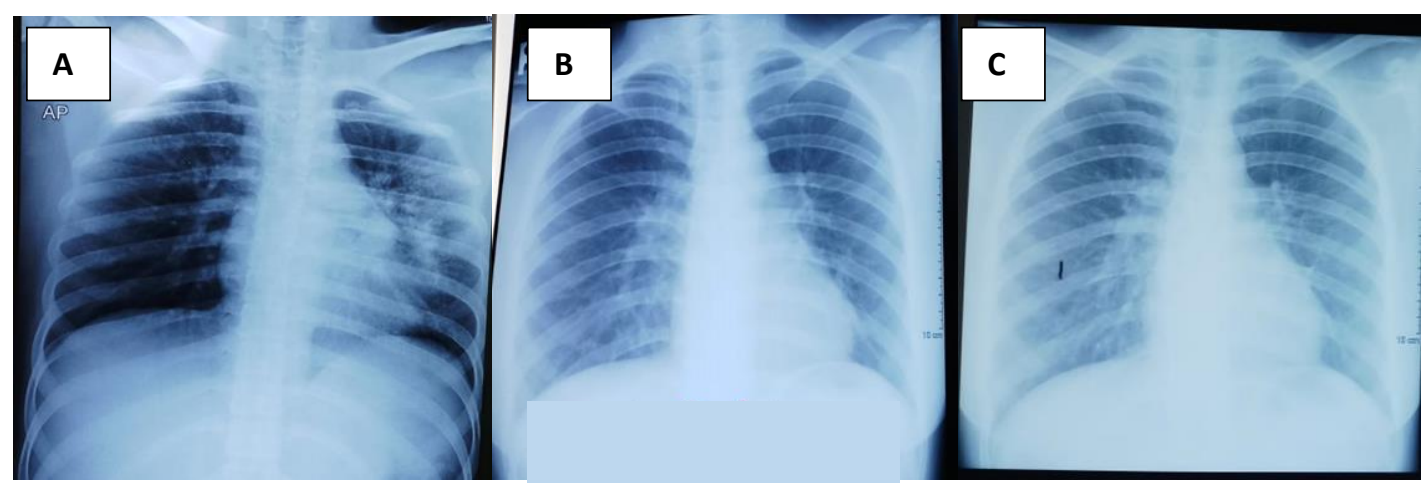

Figure 1. A. Chest radiology taken on January $18^{\text {th }} 2018$ revealed opacities in $2 / 3$ superior in the left lung before treatment;

B. Chest radiology taken on September $28^{\text {th }} 2018$ after 9 months therapy. The opacity disappeared;

C. Chest radiology taken on January $2^{\text {nd }} 2019$ after 12 months therapy. There was no opacity

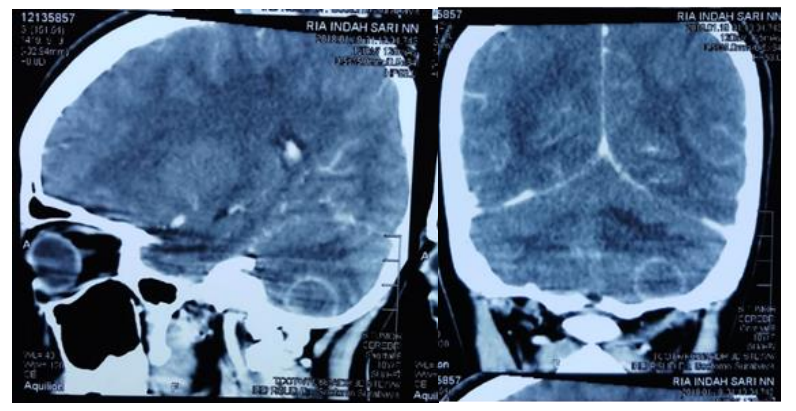

Figure 4. CT head scan with contrast taken on January $19^{\text {th }}$ 2018 showed ring contrast enhancement in left subcortical hemisphere cerebellum with size $1.8 \times 1.8 \times 1.7 \mathrm{~cm}$.

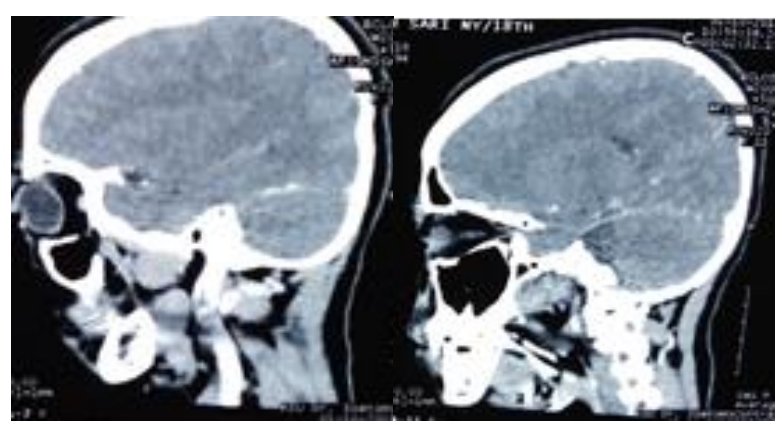

Figure 5. CT head scan with contrast taken on October $1^{\text {st }}$ 2018. After 6 months of treatment, the cerebellar tuberculoma was gone. 
Pathogenesis of cerebellar tuberculoma in this case was first M.TB infected the lungs, then it spread hematogenously to the central nervous system. Central nervous system has a lot of blood supply (rich of oxygen). ${ }^{6}$ M.TB has an ability to penetrate the blood brain barrier. In the beginning of infection, M.TB made a small lesion. This lesion in a time would get bigger, it could press the space of inner brain. The result is elevated pressure of intracranial, manifested as dizziness. This lesion could make a compression at pedunculus os temporal with the result of hemiparesis gradually. ${ }^{7,8}$ The blood brain barrier (Figure 6) is one of the defense mechanisms to protect the brain from infection. The blood brain barrier contains endothelial cells and astrocyte cells, which collaborate with macrophage that activated microglia to attack M.TB. In the experiments with rats, microglia infected by M.TB then replicated inside microglia, with result form of multinuclear giant cell. M.TB has the ability to suppress microglia at central nervous system. ${ }^{9}$ Microglia triggers the cytokine proinflammations such as TNF- $\alpha$ (Tumor Necrosis Factor Alpha), it controlled permeability of blood brain barrier to protect from infection. Astrocyte cell secreted G-CSF (Granulocyte colony-stimulating factor), and GM-CSF (Granulocyte-macrophage colonystimulating factor). G-CSF activated leukocyte and GMCSF made loss of appetite and basic metabolism decrease. Both GM-CSF and G-CSF inducted T-cells proliferation to be a Th-2 Cells and increase immunity from M.TB and resist neuron cells from death. ${ }^{9}$

Clinical symptoms of extra lung TB in central nervous systems are mostly headache, vomiting, and loss of appetite. Seizure could be a manifestation with or without fever. ${ }^{10}$ In India, clinical symptoms such as fever, headache, vomiting, loss of consciousness, and seizure were found. ${ }^{11}$ In this case, the patient complained of dizziness since 7 days before being admitted to the hospital, reduced motor function at upper and limb right extremity. The patient complained classic TB symptoms such as dry cough, loss of appetite, and night sweating.

Non-invasive diagnostic is recommended to perform rather than invasive method such as brain tissue biopsy. Microbiological examination is geneXpert M.TB from phlegm and from liquor cerebrospinal (the sensitivity is $83.1 \%$ and the specificity is $98.7 \%$, liquor cerebrospinal for geneXpert is about 8-10 ml). Smear of liquor cerebrospinal with acid-fast bacilli/Ziehl-Neelsen $(\mathrm{ZN})$ is a quick examination but the sensitivity is low (10-20\%). Liquor cerebrospinal culture has a high sensitivity (about 60-70\%), but the result comes out more than 2 weeks later. In this case, there was no sample from cerebrospinal liquor or from phlegm.
IGRA (Interferon-gamma release assay) is not recommended, the result is not specific and this method needs a lot of liquid cerebrospinal. The specificity is still unknown, but sensitivity is $90.2 \% .^{12,13}$ ADA (Adenosine deaminase) test from liquor cerebrospinal resulted positive if more than $8 \mathrm{U} / \mathrm{L}$. Decrease the amount of ADA after 1-2 weeks after treatment of TB. Nevertheless it is not recommended for TB in the central nervous system. Case study for liquor cerebrospinal explained that the sensitivity was $44 \%$ and the specificity was $71 \% .{ }^{13}$ Recommended imaging for this case was Head CT-Scan thorax without contrast (the sensitivity was $41 \%$ and the specificity was $100 \%$ ), because it was cheaper than Head MRI (the sensitivity was $86 \%$ and the specificity was $90 \%) .{ }^{14}$ Head CT Scan with contrast gave us a result as hypodensity with ring enhancement. $^{3}$

Risk factors for extra lung TB central nervous system are young age, HIV, malnutrition, history of measles, alcoholism, cancer, and using immunosuppressive agents. ${ }^{14}$ The patient had malnutrition, her BMI was $17.80 \mathrm{~kg} / \mathrm{m}^{2}$.

The therapies for extra lung TB, especially for TB in central nervous system are rifampicin, isoniazid, pyrazinamide, and ethambutol for 2 months of intensive phase. Then continued with continued phase with isoniazid, rifampicin, and ethambutol for 10-12 months. ${ }^{14}$

The pharmacology of ATD, rifampicin, and isoniazid are bactericidal drugs and penetrated to the cerebrospinal fluid. ${ }^{14}$ Streptomycin injection penetrated cerebrospinal fluid and killed M.TB in the first 14 days of therapy. ${ }^{12}$ Steroid is rarely used for the cases in central nervous system TB, steroid is recommended for encephalopathy TB and meningitis TB. Steroid works to decrease inflammation respond at meninges and subarachnoid space, decrease the edema of cerebrum and spinal cord, and decrease inflammation at microvascular. ${ }^{13,15}$ Surgery intervention is performed for central nervous system TB when there is a complication such as hydrocephalus to reduce pressure intracranial. ${ }^{1}$

\section{CONCLUSION}

Cerebellum tuberculoma is a rare presentation of active TB and is difficult to diagnose because the signs and symptoms are not specific. Tuberculoma in central nervous system should be distinguished from other diagnosis, like tumor or abscess. The diagnosis must be made quickly because there are complications such as extremity paralysis and sensory loss. Radiological imaging plays an important role in the diagnosis and monitoring of the disease. The appearance 
of tuberculoma on the patient's CT scan showed nodular enhancing lesion. Intracranial tuberculoma should be treated conservatively, since the majority of these lesions resolve completely with ATD. The patient started ATD ( $1^{\text {st }}$ category 4 FDC (Fixed Dose Combination) intensive phase for 2 months and injection of streptomycin intramuscular. The patient then continued with phase 2 FDC for 10 months) and the lesions completely disappeared within 6 months. We wanted to present our case due to well-response of ATD and being an example of atypical presentation of TB both clinically and radiologically. There was no sample from cerebrospinal liquor or from phlegm.

\section{REFERENCES}

1. Lee J. Y. (2015). Diagnosis and treatment of extrapulmonary tuberculosis. Tuberculosis and respiratory diseases, 78(2), 47-55. https://doi.org/10.4046/trd.2015.78.2.47

2. Organization WH. Global Tuberculosis Report 2018. France, https://www.who.int/tb/publications/global_report/gtbr2 018_main_text_28Feb2019.pdf (2018).

3. DeLance, A. R., Safaee, M., Oh, M. C., Clark, A. J., Kaur, G., Sun, M. Z., Bollen, A. W., Phillips, J. J., \& Parsa, A. T. (2013). Tuberculoma of the central nervous system. Journal of clinical neuroscience : official journal of the Neurosurgical Society of Australasia, 20(10) 1333-1341. https://doi.org/10.1016/j.jocn.2013.01.008

4. Turgut M, Akhaddar A, Turgut A, et al. Tuberculosis of the Central Nervous System: Pathogenesis, Imaging, and Management. 2017. Epub ahead of print 13 June 2017. DOI: 10.1007/978-3-319-50712-5.

5. Subuh M, Priohutomo S. Pedoman Nasional Penyakit TB 2014. 2014

6. Rock, R. B., Olin, M., Baker, C. A., Molitor, T. W., \& Peterson, P. K. (2008). Central nervous system tuberculosis: pathogenesis and clinical aspects. Clinical microbiology reviews, 21(2), 243-261. https://doi.org/10.1128/CMR.00042-07
7. Bhardwaj, H., Porwal, Y. C., \& Gupta, B. (2012). Cerebellar Tuberculoma Presenting as Acute Reversible Hemiplegia: A Rare Presentation. New Indian J. Surgery, 3, 218.

8. Parekh, R., Haftka, A., \& Porter, A. (2014). A rare case of central nervous system tuberculosis. Case reports in infectious diseases, 2014, 186030. https://doi.org/10.1155/2014/186030

9. Spanos, J. P., Hsu, N. J., \& Jacobs, M. (2015). Microglia are crucial regulators of neuro-immunity during central nervous system tuberculosis. Frontiers in cellular neuroscience, 9 , https://doi.org/10.3389/fncel.2015.00182

10. Thwaites, G., Fisher, M., Hemingway, C., Scott, G., Solomon, T., Innes, J., \& British Infection Society (2009). British Infection Society guidelines for the diagnosis and treatment of tuberculosis of the central nervous system in adults and children. The Journal of infection, 59(3), $167-187$. https://doi.org/10.1016/j.jinf.2009.06.011

11. Aher, A., Paithankar, M., \& Bhurke, B. (2018). Study of Central Nervous System Tuberculosis. The Journal of the Association of Physicians of India, 66(1), 41-44.

12. Raza, S., Sadaf, A., Fecto, F., Ali, R. P., Bari, M., Enam, A. (2004). Patterns of tuberculosis in the central nervous system. Infectious Diseases Journal of Pakistan, 99-104. Available at: https://ecommons.aku.edu/pakistan_fhs_ mc_mc/147

13. Schaller, M. A., Wicke, F., Foerch, C., \& Weidauer, S. (2019). Central Nervous System Tuberculosis : Etiology, Clinical Manifestations and Neuroradiological Features. Clinical neuroradiology,29(1), 3-18. https://doi.org/10.1007/s00062-018-0726-9

14. Bahr, N. C., Marais, S., Caws, M., van Crevel, R., Wilkinson, R. J., Tyagi, J. S., Thwaites, G. E., Boulware, D. R., \& Tuberculous Meningitis International Research Consortium (2016). GeneXpert MTB/Rif to Diagnose Tuberculous Meningitis: Perhaps the First Test but not the Last. Clinical infectious diseases : an official publication of the Infectious Diseases Society of America, 62(9), $1133-1135$ https://doi.org/10.1093/cid/ciw083

15. Varaine F, Rich ML. Sputum Specimen: Collection, Storage and Shipment. In: Grouzard V (ed) Tuberculosis: Practical guide for clinicians, nurses, laboratory technicians and medical auxiliaries. Geneva: Médecins Sans Frontières, pp. 212-214. 